\title{
Percutaneous Left Main Intervention
}

\author{
Afzalur Rahman, MD, FRCP, FACC \\ Dhaka, Bangladesh
}

(Cardiovasc. j. 2013; 5(2): 131-132)

Surgery with coronary artery bypass grafting (GABG) has been the standard of care for obstructive left main coronary disease for more than 3 decades. The benefits of CABG as exemplified in the Coronary Artery Surgery Study coupled with the dismal early outcomes of percutaneous treating left main disease with balloon angioplasty created a 'forbidden zone' for Interventionalists. ${ }^{1,2}$

A current literature search revealed more than 200 peer reviewed citations in the past 30 months (including more than half a dozen editorials in major cardiology journals) dedicated to the subject of left main percutaneous coronary intervention $(\mathrm{PCl})$. Much of these data created new enthusiasm for left main intervention with drug eluting stents (DES), reinforced by metaanalysis of randomized trials indicating equivalent 5 to 10 year mortality rate and myocardial infarction in patients undergoing multivessel PCI compared with GABG. ${ }^{3,4}$

Favorable recent studies of left main DES include single-center registries, ${ }^{5}$ propensitymatched cohorts compared with $\mathrm{CABG},{ }^{6}$ metaanalysis of multicenter registries ${ }^{7,8}$ and most importantly the 705 patient left main subset analysis of the SYNTAX (Synergy Between Percutaneous Coronary Intervention with Taxus and Cardiac Surgery) randomized trial. ${ }^{9}$ The diversity and richness of these data sets are themselves a clear indication that left main PCI is now in widespread clinical practice across the globe.

LE MANS (Unprotected Left Main Stenting Versus Bypass Surgery) Registry collaborators also showed outcomes of left main PCI in an 11year collaborative effort that began in 1997 in a single country. ${ }^{10}$ Certain conclusions can be drawn from their observations: l) left main stenting in skilled hands can be performed with low morbidity and mortality with no late "catch- up", 2) DES have improved clinical outcomes compared with bare metal stents, principally by substantially reducing the need for repeat revascularization, 3) the rates of early, late and very late thrombosis are low despite the limited use of dual anti-platelet therapy after 2 years with both stent types and 4) long-term survival after left main stenting is excellent in those patients with left main disease without concomitant 3 vessel disease.

The power of these observations is somewhat limited by a lack of randomization against surgery which is evaluated by the SYNTAX trial in which Paclitaxel-eluting stents compared with CABG in patients with left main disease and either lowor moderate risk coronary artery anatomic complexity resulted in comparable or lower rates of composite major adverse cardiovascular events.

The SYNTAX (Synergy between Percutaneous Coronary intervention with TAXUS and Cardiac Surgery) trial was the first randomized clinical trial to compare clinical outcomes in patients with multivessel and left main CAD. ${ }^{2}$ ln the SYNTAX study, the SYNTAX score (based on angiographic characteristics) provided powerful discrimination of clinical outcomes in all patients undergoing revascularization. ${ }^{3}$ This score represents the most comprehensive anatomic characterization of CAD to date and has become the subject of intense clinical Investigation. Patients with a low SYNTAX score had low rates of nonfatal myocardial infarction or death during study follow-up; and patients with the highest tertile of SYNTAX score had the worst clinical outcomes at study end. A post hoc evaluation of the SYNTAX trial by the study investigators revealed that the SYNTAX score was as predictive or had a more predictive value than the Euro SCORE (European System for Cardiac Operative Risk Evaluation), a risk scoring system 
based on baseline clinical characteristics. ${ }^{4} \mathrm{~A}$ risk scoring based on angiographic characteristics alone was more predictive of clinical outcomes than one based on well established clinical characteristics has surprised many and spurred interest in evaluating the SYNTAX score in populations beyond the SYNTAX trial itself. ${ }^{4,5}$ Some investigators assessed 2 risk scores combining both angiographic and clinical characteristics: the global risk classification, using both the SYNTAX and EUROSCORE to create tertiles of low- to high risk and the clinical SYNTAX score, derived by multiplying the SYNTAX score by the age, creatinine, ejection fraction (AGEF) score. ${ }^{7,8}$ These scores as well as the individual angiographic and clinical scores were compared for performance with 2 metrics: calibration and discrimination. Distilling multiple results and comparisons from this registry, the authors concluded that the global risk classification score had the overall best calibration and discrimination of the various scores in the PCI patients, whereas the AGEF score provided the best overall performance in the CABG patients. Interestingly, only a high SYNTAX score $\geq 32$ was able to identify a better outcome with 1 revascularization strategy compared with the other (in this case GABG vs. PCI) among all the scores evaluated.

The weight of evidence supports the position that: 1) left main PCI with DES of the ostium or shaft can be performed with very low morbidity and mortality and with very low rates of repeat revascularization, 2) stenosis of the distal left main can be effectively treated with a single 'crossover' stent in the majority of cases and has become the current preferred strategy, 3) stent thrombosis of the left main segment is infrequent and 4) PCI with DES will result in non inferior outcomes to CABG in many patients with unprotected left main disease, although selected patients with very complex and for triple-vessel disease still benefit from a primary surgical approach.

\section{References:}

1. Chaitman BR, Fisher LD, Bourassa MG et al. Effect of coronary bypass surgery on survival patterns in subsets of patients with left main coronary disease; report of the Collaborative Study in Coronary Artery Surgery (CASS). Am J Cardiol 1981;48:765-777.

2. O'Keefe JH Jr., Hartzier GO, Rutherford BD et al. Left main coronary angioplasty: early and late results of 127 acute and elective procedures. Am J Cardiol 1989; 64: 144-147.

3. Hiatky MA, Boothroyd DB, Bravata DM et al. Coronary artery bypass Surgery compared with percutaneous coronary interventions for multivsssel disease: a collaborative analysis of individual patient data from ten randomized trials. Lancet 2009;373:1190-1197.

4. Daemen J, Boersma E, Flather M et al. Long term safety and efficacy of percutaneous coronary intervention with stenting and coronary artery bypass surgery for multivessel coronary artery disease: a metaanalysis with 5-year patient-level data from the ARTS, ERACI-II, MASSII and SoS trials. Circulation 2008;118:1146-1154.

5. Chieffo A, Stankovic G, Bonizzoni E et al. Early and midterm results of drug-Eluting stent implantation in unprotected left main. Circulation 2005;111: 791-795.

6. Seung KB, Park DW, Kim YH et al. Stents versus coronary artery bypass grafting for left main coronary artery disease. N Eng J Med 2008; 155:274-283.

7. Biondi-Zoccai GG, Lotrionte M, Moretti C et al. A collaborative systematic review and meta-analysis on 1278 patients undergoing percutaneous drug-eluting stenting for unprotected left main coronary artery disease. Am Heart J 2007;155:274-283.

8. Chieffo A, Park SJ, Valgimigli M et al. Favorable longterm outcomes after drug-eluting stent implantation in non-bifurcation lesions that involve unprotected left main coronary artery: a multicenter registry. Circulation 2007;116:158-163. 